\title{
EFFICIENT GAUSSIAN INFERENCE ALGORITHMS FOR PHASE IMAGING
}

\author{
Zhong Jingshan ${ }^{1}$, Justin Dauwels ${ }^{1}$, Manuel A. Vázquez ${ }^{2 *}$, Laura Waller ${ }^{3}$ \\ ${ }^{1}$ Nanyang Technological University, School of Electrical and Electronic Engineering, Singapore 639798 \\ ${ }^{2}$ Universidad Carlos III de Madrid, Depto. de Teoría de la Señal y Comunicaciones, Madrid, Spain. \\ ${ }^{3}$ Princeton University, Department of Electrical Engineering, Princeton, NJ 08544 US
}

\begin{abstract}
Novel efficient algorithms are developed to infer the phase of a complex optical field from a sequence of intensity images taken at different defocus distances. The non-linear observation model is approximated by a linear model. The complex optical field is inferred by iterative Kalman smoothing in the Fourier domain: forward and backward sweeps of Kalman recursions are alternated, and in each such sweep, the approximate linear model is refined. By limiting the number of iterations, one can trade off accuracy vs. complexity. The complexity of each iteration in the proposed algorithm is in the order of $N \log N$, where $N$ is the number of pixels per image. The storage required scales linearly with $N$. In contrast, the complexity of existing phase inference algorithms scales with $N^{3}$ and the required storage with $N^{2}$. The proposed algorithms may enable real-time estimation of optical fields from noisy intensity images.
\end{abstract}

Index Terms - Phase imaging, Kalman filter

\section{INTRODUCTION}

When coherent (laser) light passes through or reflects from an object, its intensity and phase are altered as it continues to propagate. Phase perturbations contain important information about the object; for example, transparent biological cells are invisible in a focused microscope, but impart distinct phase changes. However, only the intensity can be measured directly, as phase oscillates far too quickly, and it needs to be reconstructed computationally. This "phase problem" of optical imaging has been around for decades, but only recently has been posed as an inference problem [1,7], in principle allowing for experimental noise in the images. However, most phase reconstruction methods are ad hoc and ignore the important effects of noise.

Traditional methods for recovering phase involve complicated interferometric setups, so there is a significant experimental advantage to methods such as ours, which solve

${ }^{*}$ M. A. Vázquez acknowledges the support of the Ministry of Education of Spain(Programa Nacional de Movilidad de Recursos Humanos del Plan Nacional de I-D+i 2008-2011). He conducted this research while visiting the School of Electrical and Electronic Engineering at NTU. for phase from a set of intensity images captured at various distances along the optical axis. The first such method, the Gerchberg-Saxton(GS) method [2,3], treats the problem as convex (which it is generally not) and iterates back and forth between two domains to reduce error at each iteration; it is strongly sensitive to the noise in the last image. The direct method $[4,5,6]$ exploits the Transport of Intensity Equation (TIE); it is based on first- and higher-order derivatives, and it is not robust to noise. A few statistical approaches have been proposed as well; an approximation to the maximum likelihood estimator is derived in $[7,8]$. However, it easily gets stuck in local maxima, and sometimes leads to poor results. In [1] an extended Kalman filter was used to solve for phase with significant noise corruption. However, the memory requirements are of order $N^{2}$, which is unfeasible for practical image sizes of multiple megapixels, and the long computation times are impractical for real-time applications.

In this paper we introduce a novel approach to the problem of amplitude and phase estimation. Just like the method of [1], it is based on the Kalman filter. However, we make certain convenient approximations to significantly reduce the computational complexity, without jeopardizing the quality of the reconstruction. Moreover, it is iterative and can trade off computational complexity vs. reconstruction accuracy.

This paper is organized as follows. In Section 2, a brief description of the problem and the mathematical model is presented. We derive our algorithm in Section 3. We present experimental results in Section 4, and we offer concluding remarks in Section 5.

\section{PROBLEM DESCRIPTION AND STATE SPACE MODEL OF THE OPTICAL FIELD}

We aim at estimating the 2D complex-field $A\left(x, y, z_{0}\right)$ at the focal plane $z_{0}$, from a sequence of noisy intensity images $I(x, y, z)$ captured at various distance $z_{0}, z_{1}, z_{2}, \ldots, z_{N}$. We assume a linear medium with homogenous refractive index and coherent (laser) illumination, such that the complexfield at $z_{0}$ fully determines the complex-field at all other planes. The complex optical field at $z$ is $A(x, y, z)=$ $|A(x, y, z)| e^{i \phi(x, y, z)}$, where $|A(x, y, z)|$ is the amplitude, and $\phi(x, y, z)$ is the phase. Propagation is modeled by the 
homogeneous paraxial wave equation

$$
\frac{\partial A(x, y, z)}{\partial z}=\frac{i \lambda}{4 \pi} \nabla_{\perp}^{2} A(x, y, z)
$$

where $\lambda$ is the wavelength of the illumination, and $\nabla_{\perp}$ is the gradient operator in the lateral $(x, y)$ dimensions only. The noisy measurements $I(x, y, z)$ usually adhere to a (continuous) Poisson distribution:

$p[I(x, y, z) \mid A(x, y, z)]=e^{-\gamma|A(x, y, z)|^{2}} \frac{\left(\gamma|A(x, y, z)|^{2}\right)^{I(x, y, z)}}{I(x, y, z) !}$

where $\gamma$ is the photon count detected by the camera. The measurement at each pixel $I(x, y, z)$ is assumed statistically independent of any other pixel (conditioned on the optical field $A(x, y, z))$.

We can discretize the optical field $A(x, y, z)$ as a rasterscanned complex column vector $\mathbf{a}_{n}$, and similarly discretize the measurement $I(x, y, z)$ as column vector $\mathbf{I}_{n}$. We denote by $b(u, v, z)$ the 2-D Fourier transform of $A(x, y, z)$. The column vector $\mathbf{b}_{n}$ is again raster-scanned from $b(u, v, z)$, and hence can be expressed as $\mathbf{b}_{n}=\mathbf{K} \mathbf{a}_{n}$, where $\mathbf{K}$ is the discrete Fourier transform matrix. Since $\mathbf{K}$ is unitary, we can write $\mathbf{K} \mathbf{K}^{H}=\mathbf{K}^{H} \mathbf{K}=\mathbf{U}$ (with normalization), where $\mathbf{U}$ is the identity matrix and $\mathbf{K}^{H}$ denotes the hermitian of $\mathbf{K}$.

We can define the propagation matrix at $z_{n}$ as [9]:

$$
\begin{gathered}
\mathbf{H}_{n}=\operatorname{diag}\left(\exp \left[-i \lambda \pi\left(\frac{u_{1}^{2}}{L_{x}^{2}}+\frac{v_{1}^{2}}{L_{y}^{2}}\right) \Delta_{n} z\right], \ldots,\right. \\
\left.\quad \exp \left[-i \lambda \pi\left(\frac{u_{M}^{2}}{L_{x}^{2}}+\frac{v_{N}^{2}}{L_{y}^{2}}\right) \Delta_{n} z\right]\right),
\end{gathered}
$$

where $L_{x}$ and $L_{y}$ are the width and height of the image.

The relation between two images with distance $\Delta_{n} z$ in Fourier domain can be written as,

$$
\mathbf{b}_{n}=\mathbf{H}_{n} \mathbf{b}_{n-1} \text {. }
$$

We approximate the Poisson observation model (2) with a Gaussian distribution of same mean and covariance. In particular, we consider the approximate observation model:

$$
\mathbf{I}_{n}=\gamma \operatorname{diag}\left(\mathbf{a}_{n}^{*}\right) \mathbf{a}_{n}+\mathbf{v}
$$

where $\mathbf{v}$ is a vector of Gaussian components with zero mean and covariance $\mathbf{R}=\gamma \operatorname{diag}\left(\mathbf{a}_{n}^{*}\right) \operatorname{diag}\left(\mathbf{a}_{n}\right)$.

In summary, a simplified state-space model for the problem at hand is:

$$
\text { state : } \mathbf{b}_{n}=\mathbf{H}_{n} \mathbf{b}_{n-1}
$$

observation : $\mathbf{I}_{n}=\mathbf{J}\left(\mathbf{b}_{n}\right) \mathbf{b}_{n}+\mathbf{v}$, with $\mathbf{v} \sim(0, \mathbf{R})$,

where $\mathbf{J}\left(\mathbf{b}_{n}\right)=\gamma \operatorname{diag}\left(\mathbf{K}^{T} \mathbf{b}_{n}^{*}\right) \mathbf{K}^{H}$.

\section{STATE ESTIMATION BY APPROXIMATE KALMAN FILTERING}

A modified Kalman filter for the state-space model (6)-(7) is now derived. Since the observation equation (7) is non-linear, the resulting algorithm is suboptimal. However, the resulting estimates of $b_{n}$ are accurate enough for our purposes, and the simplicity of the filter makes it amenable for real-time implementation. From the equations of the standard Kalman filter [10], we have the three following steps:

(1) Initialization: $\mathbf{b}_{0}$ and error covariance matrix.

(2) Prediction: $\widehat{\mathbf{b}_{n}}=\mathbf{H b}_{n-1} ; \widehat{\mathbf{M}_{n}}=\mathbf{H M}_{n-1} \mathbf{H}^{H}$.

(3) Update:

$$
\begin{aligned}
\mathbf{G}_{n} & =\widehat{\mathbf{M}_{n}} \mathbf{J}^{H}\left(\widehat{\mathbf{J}} \widehat{\mathbf{M}_{n}} \mathbf{J}^{H}+\mathbf{R}\right)^{-1} \\
\mathbf{b}_{n} & =\widehat{\mathbf{b}_{n}}+\mathbf{G}_{n}\left(\mathbf{I}_{n}-\widehat{\mathbf{J} \mathbf{b}_{n}}\right) \\
\mathbf{M}_{n} & =\widehat{\mathbf{M}_{n}}-\mathbf{G}_{n} \mathbf{J} \widehat{\mathbf{M}_{n}}
\end{aligned}
$$

The computational burden of the above algorithm can be reduced significantly by constraining the state covariance matrix to be diagonal. We will show that in order for this condition to hold at all times, it suffices to initialize the algorithm with a diagonal covariance matrix (that is, $\mathbf{M}_{0}=\alpha_{0} \mathbf{U}$ ). With this choice of initialization, Theorem 1 provides a simple update rule for the covariance matrix $\mathbf{M}_{n}$, whereas Theorem 2 provides a simplified expression for $\mathbf{b}_{n}$. In order to prove those theorems, we have first derived two simple lemmas (Lemma 1 and 2). For the sake of brevity, we omit all proofs here.

Lemma 1. If $\mathbf{M}_{n-1}$ is diagonal, then $\widehat{\mathbf{M}_{n}}=\mathbf{H M}_{n-1} \mathbf{H}^{H}=$ $\mathbf{M}_{n-1}$.

Lemma 2. $\mathbf{R}=\gamma^{-1} \mathbf{J} \mathbf{J}^{H}$.

Theorem 1. If $\mathbf{M}_{0}=\alpha_{0} \mathbf{U}$, then $\mathbf{M}_{n}=\alpha_{n} \mathbf{U}$ with $\alpha_{n}=$ $\frac{\alpha_{n-1}}{\gamma \alpha_{n-1}+1}$.

Theorem 2. The update formula for state is

$$
\mathbf{b}_{n}=\left(1-\alpha_{n} \gamma\right) \widehat{\mathbf{b}_{n}}+\alpha_{n} \gamma \mathbf{J}^{-1} \mathbf{I}_{n} .
$$

Table 1. Low-Complexity Algorithm for Inferring an Optical Field.

$$
\begin{aligned}
& \text { (1) Initialization: } \mathbf{b}_{0} \text { and error covariance ma- } \\
& \text { trix } \mathbf{M}_{0}=\alpha_{0} \mathbf{U} \text {. } \\
& \text { (2) Prediction: } \mathbf{b}_{n}=\mathbf{H b}_{n-1} ; \widehat{\mathbf{M}_{n}}=\mathbf{M}_{n-1} \text {. } \\
& \text { (3) Update: } \\
& \quad \widehat{\mathbf{a}_{n}}=\mathbf{K}^{H} \widehat{\mathbf{b}_{n}} \\
& \quad \mathbf{M}_{n}=\alpha_{n} \mathbf{U} \text {, with } \alpha_{n}=\frac{\alpha_{n-1}}{\gamma \alpha_{n-1}+1} \\
& \quad \mathbf{b}_{n}=\left(1-\alpha_{n} \gamma\right) \widehat{\mathbf{b}_{n}}+\alpha_{n} \gamma \mathbf{J}^{-1} \mathbf{I}_{n}
\end{aligned}
$$

The resulting algorithm is summarized in Table 1 . Note that the inverse of $\mathbf{J}$ in equation (13) can be computed efficiently by means of a Fast Fourier Transform (FFT). Thus, if 
$N_{z}$ images are available and the size of each image is $N=$ $N_{x} \times N_{y}$, then the overall computational complexity of the proposed algorithm is $\mathcal{O}\left(N_{z} N \log (N)\right)$.

\section{EXPERIMENTAL RESULTS}

In order to assess the performance of the proposed method, two sets of images have been considered. Data set 1 consists of 100 images of size $100 \times 100$ pixels artificially generated to simulate a complex field propagating from focus in $0.5 \mu \mathrm{m}$ steps over a distance of $50 \mu \mathrm{m}$ with illumination wavelength of $532 \mathrm{~nm}$. Pixels are corrupted by Poisson noise so that, on average, each pixel detects $\gamma=0.998$ photons. Data set 2 comprises 50 images of size $150 \times 150$ pixels acquired by a microscope. The wavelength was again $532 \mathrm{~nm}$, and the defocused intensity images were captured by moving the camera axially with a step size of $2 \mu \mathrm{m}$ over a distance of $100 \mu \mathrm{m}$.

\subsection{Data Set 1 (simulated data)}

Since the method of [1] has the computational complexity of $\mathcal{O}\left(N_{z} N^{3}\right)$ and strict storage requirements, each image has been divided into 4 blocks of size $50 \times 50$ each. Nevertheless, this approach still takes 13562.8 seconds, and is therefore impractical. The complexity of our method is $\mathcal{O}\left(N_{z} N \log N\right)$, and hence it is not necessary to divide the image. Indeed, the proposed method takes only 0.30 seconds to process the 100 (full) images.

Fig. 1(a) shows the intensity and phase images recovered by both the proposed method and that of [1], along with the true intensity and phase images. The average (across all pix-

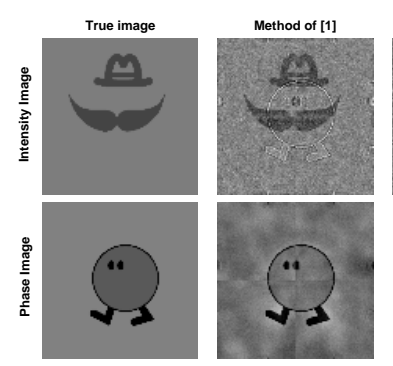

(a)
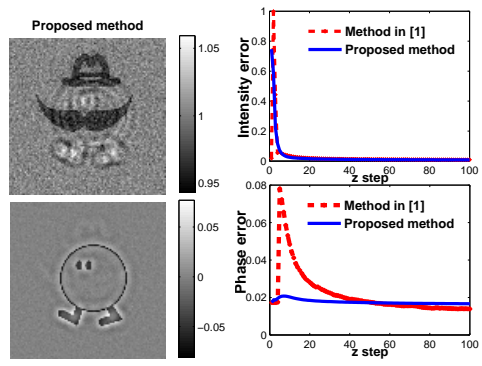

(b)
Fig. 1. (a) Recovered intensity and phase image by proposed method (Data Set 1), compared with method of [1]. (b) Intensity error and phase error for Data Set 1 , as a function of the number of images (total $=100$ ).

els) root mean square error (RMSE) of our method as compared to that of [1] is given in Table 2, with all phase results in units of radians. The two methods clearly lead to artifacts in both the intensity and phase recovered images: traces of the phase image can be seen in the intensity image and vice
Table 2. Comparison with the method in [1].

Complexity Time [s] Storage Intensity/Phase Error Block

$\begin{array}{cccccc}\text { Method of [1] } & \mathcal{O}\left(N_{z} N^{3}\right) & 13562.8 & \mathcal{O}\left(N^{2}\right) & 0.0091 / 0.01388 & \text { Yes } \\ \text { Proposed method } \mathcal{O}\left(N_{z} N \log N\right) & 0.30 & \mathcal{O}(N) & 0.0079 / 0.01664 & \text { No }\end{array}$

versa. As we will show in Section 4.3, those traces can be removed through an iterative algorithm.

It can also be observed in Fig. 1(a) that the images recovered by the method of [1] exhibit a block effect that shows artifacts in the form of straight lines crossing the images. This is due to the latter being processed in segments rather than as a whole. The proposed method can handle the entire images efficiently and hence, no segmentation artifacts occur.

In both the proposed method and that of [1] the estimation error decreases as images are processed, as illustrated in Fig. 1(b), which compares the RMSE incurred for increasingly more images being added. Ultimately, both methods seem to converge to to similar estimation errors for both the intensity and phase. However, our low-complexity method converges faster and uses significantly less memory.

Table 2 summarizes the results for the proposed method and that of [1] for Data Set 1.

\subsection{Data Set 2 (experimental data)}

Fig. 2 shows the intensity and phase images estimated by the proposed method when using Data Set 2, which was collected from the experimental data. Running the algorithm over this data set took 0.32 seconds whereas the algorithm of [1] took 7969.52 seconds, with blocks of size $40 \times 40$. The recovered intensity and phase images did not exhibit a block effect, but had some phase errors inside the lettering of MIT. As we will show in next section, the results can be further improved by means of an iterative algorithm.

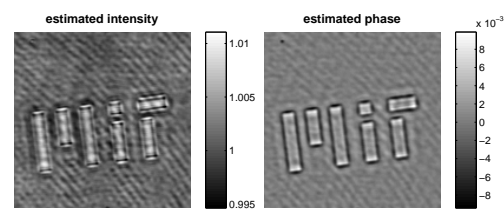

Fig. 2. Estimated intensity and phase by proposed method for Data Set 2.

\subsection{Iterative Algorithm}

Instead of carrying out one forward sweep of Kalman recursions, we now alternate forward and backward sweeps multiple times. This will allow us to trade off computational complexity for accuracy. When the forward recursion ends at the last image, a backward recursion is started, initialized by the current estimate of the (last) image. Likewise, when the backward recursion ends at the first image, a forward recursion is 
started with the current estimate of the first image as initialization.

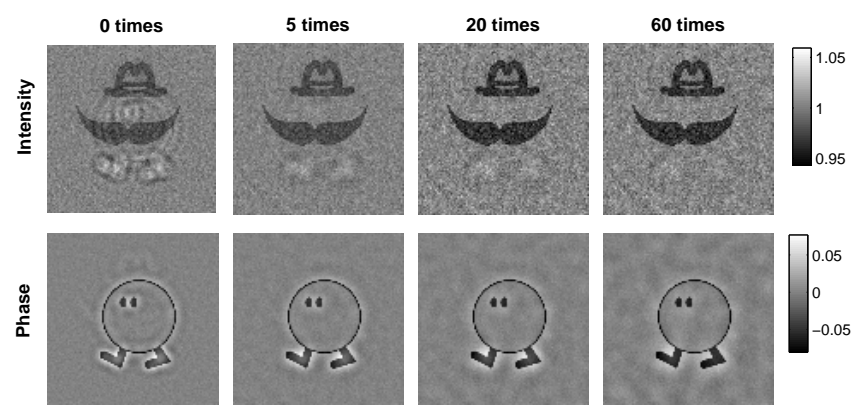

Fig. 3. Estimated intensity and phase by proposed method for Data Set 1 at iterations 0, 5, 20 and 60, with computational time of $0.30 \mathrm{~s}, 3.18 \mathrm{~s}, 12.11 \mathrm{~s}$, and $37.04 \mathrm{~s}$ respectively.

Table 3. RMS for iterative method

\begin{tabular}{lllll} 
Number of Iterations & 1 & 5 & 20 & 60 \\
\hline
\end{tabular}

RMS for Intensity Image $0.0078 \quad 0.0072 \quad 0.00710 .0071$

RMS for Phase Image 0.01650 .01580 .01520 .0147

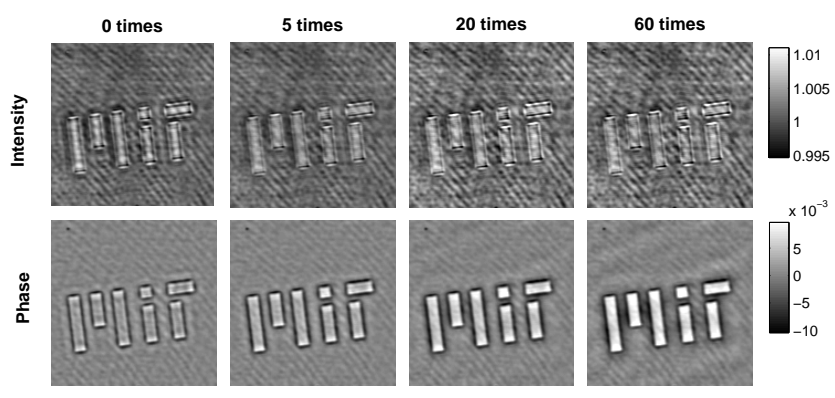

Fig. 4. Estimated intensity and phase by proposed method for Data Set 2 at iterations 0, 5, 20 and 60, with computational time $0.32 \mathrm{~s}, 3.39 \mathrm{~s}, 12.90 \mathrm{~s}$, and $39.50 \mathrm{~s}$ respectively.

The recovered intensity and phase images for Data Set 1 and different numbers of iterations are displayed in Fig. 3. Each iteration consists of a forward and backward sweep of the Kalman recursions. It shows how the artifacts gradually vanish as the number of iterations increases. This is also assessed numerically in Table 3, which shows the RMSE of the intensity and phase images recovered for different numbers of iterations. The difference in the RMSE is small but the image quality improvements can be appreciated by comparing the reconstructed images in Figs. 3 and 4.

Fig. 4 shows the recovered phase and intensity images for the Data Set 2 with different numbers of iterations. It can be seen that the pixels of the MIT logo in the recovered phase images look brighter after a few iterations.

The computational complexity increases linearly with the number of iterations. The total computational time is on the order of tens of seconds, which is still rather low.

\section{CONCLUSIONS}

We have proposed Gaussian inference algorithms to recover the phase and amplitude of a complex optical field from noisy defocused intensity images. It requires vastly less storage than existing methods (linear vs. quadratic), and it is substantially less complex (close to linear vs. cubic), while attaining high reconstruction accuracy. Moreover, it allows to trade off between complexity and accuracy. Due to the scalability of the wave equations and the simplicity of the measurement technique, this method could find use in phase imaging beyond optical wavelengths (for example, X-ray or neutron imaging), where high-quality images are difficult to obtain and noise is significant and unavoidable.

\section{REFERENCES}

[1] L. Waller, M. Tsang, S. Ponda, and G. Barbastathis. "Complex-field estimation by Kalman filtering," Opt. Express, 19(3):2805-2815, 2011.

[2] R. Gerchberg and W. Saxton. "A practical algorithm for the determination of phase from image and diffraction plane picture," Optik, 35:273-246, 1972.

[3] J. Fienup. "Phase retrieval algorithms: a comparison," Appl. Opt., 21, 1982.

[4] M. Teague. "Deterministic phase retrieval: a Green's function solution," J. Opt. Soc. Am. A, 73(11):1434, 1983.

[5] M. Soto and E. Acosta. "Improved phase imaging from intensity measurements in multiple planes," Appl. Opt., 46(33):7978-7981, 2007.

[6] L. Waller, L. Tian, and G. Barbastathis. "Transport of intensity phase-amplitude imaging with higher order intensity derivatives," Opt. Express, 18(12):12552-12561, 2010.

[7] R. Paxman, T. Schulz, and J. Fienup. "Joint estimation of object and aberrations by using phase diversity," $J$. Opt. Soc. Am. A, 9(7):1072-1085, 1992.

[8] R. Paxman and J. Fienup. "Optical misalignment sensing and image reconstruction using phase diversity," $J$. Opt. Soc. Am. A, 5(6):914-923, 1988.

[9] J. Goodman, Introduction to Fourier Optics, McGrawHill.

[10] R. Kalman et al. "A new approach to linear filtering and prediction problems," J. basic Eng. , 82(1): 35-45, 1960. 